\title{
Spatial Georeferencing of the AutoCAD Map by Using GIS Software
}

\author{
Sabah Hussein Ali \\ Remote Sensing Center \\ University of Mosul
}

Received
$0 \bullet / 0 \checkmark / 2008$
Accepted

$10 / 09 / 2008$

\section{الخلاصة}

أصبحت تقتيـة تبـادل المعلومات ذي أهميـة كبيرة في ظهور الحاجـة إلى تهيئة كوادر

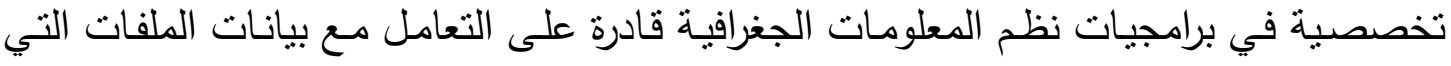

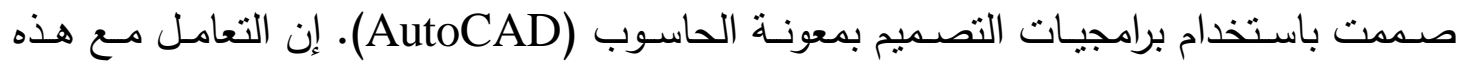

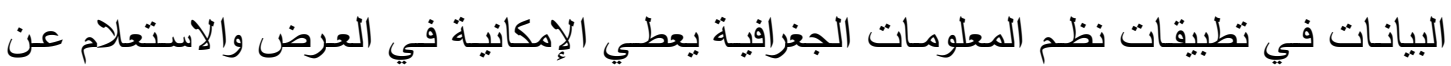

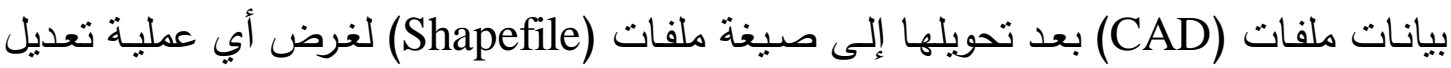

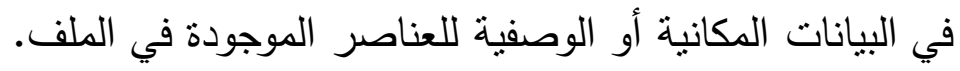

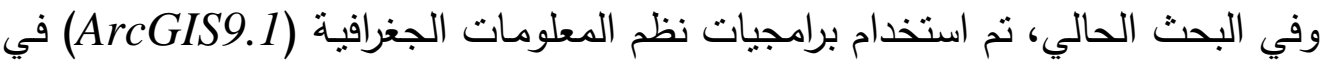

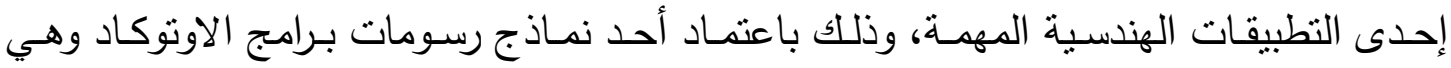
أنظمـة التصـيم بمعونـة الحاسـوب (AutoCAD) وتحويلها إلى صيخة ملفـات (Shapefile)

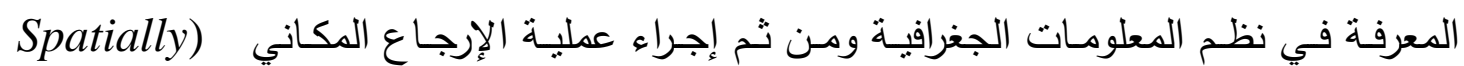

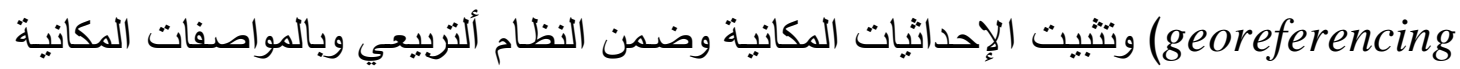

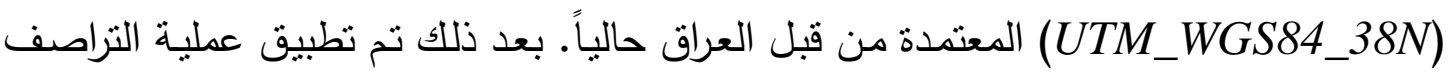
المكاني (Spatially Alignment) بين ملفات الاوتوكاد الجديدة مع بيان فضائي لنفس منطقة النموذج المعتمد في الدراسة.

إن المنهجية المستخدمة في الدراسة الحالية، نم تطبيقها لنموذج خاص يمثل حرم جامعة

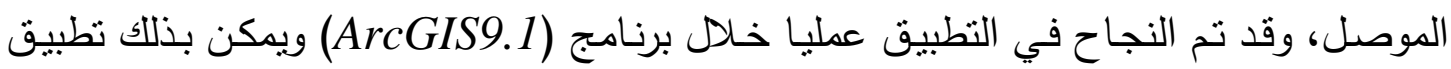

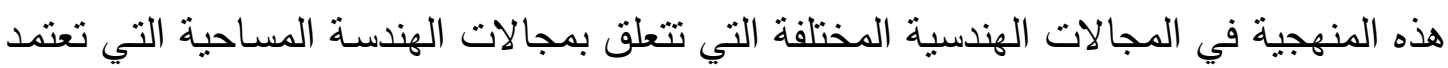
برامجيات الاوتوكاد في أساساً لها.

وقد أظهرت النتائج تطابق تام بين المضلعات بالصيغة (shapefile) مع نظيراتها التي لئي

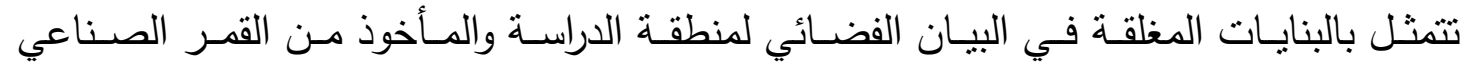

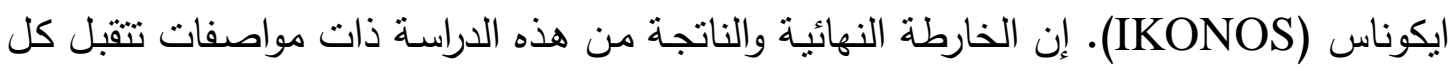

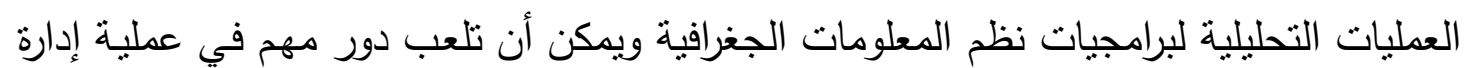
اتخاذ القرار ضمن الفقرات التي تحنويها منطقة الدراسة. 


\begin{abstract}
With the ever increasing need for design and GIS professionals to share data, it is critical that GIS users understand and are able to make use of data created with Autodesk's Computer Aided Drafting (AutoCAD) software. In GIS applications, it can be display and query the data as it exists in its native CAD file format, or it can convert it to a geodatabase feature class or shapefile if you want to edit the spatial features or attribute table.

This paper presents a procedures to using AutoCAD Data in ArcGIS by applying (.dwg) format and integrating it into standard ESRI data formats (shapefiles). The final output results of this study are a files in a GIS shapefile format that spatial georeferencing with respect to UTM WGS84_38N projected coordinate systems (metric). Spatially displacement process was applied to align the final reprojected shapefile with the reference image satellite acquired by IKONOS satellite. The satellite image covered the same area of CAD drawing (represented Mosul University Campus).

The results show, perfect coincidence between the new polygon shapefile with the closed buildings appears in the IKONOS image satellite of the study area. The output map layer was ready to be fully usable in all GIS operations and can be help in making important management decisions.
\end{abstract}

Keywords: CAD, GIS, georeferencing, geodatabase, shapefile.

\title{
Introduction
}

With the growing popularity of Geographical Information System (GIS) and the increasing availability of a wide range of datasets through the remote sensing techniques, GIS is becoming a common tool for all disciplines of the applied and theoretical researches in the world. The convergence of GIS with allied technologies, those of surveying, remote sensing, air photos, Global Positioning System (GPS) measurements, mobile computing and communications has fed a spectacular growth of these technologies [1].

In the concept of GIS, ArcGIS software sees CAD files as collections of ArcGIS feature classes not as CAD drawings. On the disk they are still CAD files, but ArcGIS doesn't see them that way. ArcGIS sees CAD files as collections of GIS objects that have a table of attributes. CAD files are not stored as feature classes with tables of attributes, but ArcGIS builds a view of the CAD file in memory where it applies assumptions and applies constraints to the CAD data to turn it directly into GIS data [2].

However, to get accurate analysis results from the CAD (Computer Aided Drafting) database through the GIS software, first it is necessary to represent the data by the real-world features, this can be applied by 
applying the georeferencing process on the database according to their spatial coordinate [3]. In this case, you have the ability to built a geodatabase feature class or shapfile ((a shapefile stores nontopological geometry and attribute information for the spatial features in a data set, the geometry for a feature is stored as a shape comprising a set of vector coordinates [4]) that enable the user to edit the spatial feature or attribute record, in the other word, simple ability to select CAD features by attribute and convert them to a new GIS feature class to highly complex geoprocessing models that automate a variety of CAD/GIS translation operations. The concept of geodatabase is used to refer to a storage format which has the ability to store the feature coordinate and attribute information in one database, that means; it is centralized spatial and attribute storage.

This paper describes a technique for clean and accurate conversions of (CAD) ".dwg" files to aligned (GIS) shapefiles ".shp" data format. The process was carried out by using a ".dwg" file sample covered the total area of the Mosul University campus. Then, by using the ArcGIS9.1, the CAD drawings were reprojected (spatial georeferencing) into a new coordinate system, Universal Transverse Mercator: UTM with respect to World Geodetic System:WGS84 at the Zone_38N. The axes of the final map as stabilized according to the UTM_WGS84_38Z. This system has been commonly used in IRAQ at the present time.

The resulting GIS format allows the user to perform many processes such as create, edit, analysis, query, delete, and display. Also, it can be used as a reference guide for the conversion process of the available CAD drawings with a large scale related to the landscape or architectural projects (or drawings) at the University or municipality sites in our province to an accurate GIS data which was represented the large scale format too.

\section{GIS and CAD data format}

GIS can be defined as an information system that is designed to work with data referenced by spatial or geographic coordinates system. In other words, a GIS is both a database system with specific capabilities for spatially-referenced data (means link the data to a geographic map), as well as a set of operations for working with the data [1]. It can be seen as automated systems for capture, storing, retrieval, analysis, and display of spatial data. These are of course, computer tools, and a GIS can then be thought of as a software package containing the elements necessary for working with spatial data.

CAD drawings are simply that - drawings - and contain no additional attributes. All CAD drawing elements are either points, polygons, or polylines (made up of a continuous string of lines). A CAD drawing element contains only the information needed to draw itself lineweight (thickness), linetype (continuous, dotted, dashed), color, and 
the layer to which it is assigned. Each individual feature class within $\mathrm{CAD}$ has an associated attribute table, usually (Drawing Exchange Format: dxf) file [5]. This table displays the original attributes from the $\mathrm{CAD}$ file. Also, $\mathrm{CAD}$ features are organized by layers, which can contain points, polylines, polygons, and annotation (as compared with a GIS shapefile, which can only contain one feature type).Each drawing feature belongs to a specific layer. In this way elements of similar type can be viewed, hidden, frozen, moved, and edited together. The CAD digital drawings are typically very accurate to large - scale and are often based on a ground survey of the site done by a certified professional surveyor.

GIS, on the other hand, has an additional functionality- its features can hold enormous amounts of data describing the features (for example, a polygon representing a house could contain information about the owner, street address, numbers of bathrooms, bedrooms, etc). Each feature has its attribute table.

Moreover, GIS data is "spatially informed" regarding adjacency and other spatial relationships. Simply put, CAD is a drawing, and GIS is a spatial database. Therefore, by turning the CAD drawings into GIS data, the fully functionally of GIS can be used to analyze and compare the data from the CAD drawings into GIS data.

\section{Georeferencing Process and Coordinate System}

In GIS concepts, raster data is commonly obtained by scanning maps or collecting aerial photographs and satellite images. Scanned map datasets don't normally contain spatial reference information, either embedded in the file or as a separate file. With aerial photography and satellite imagery, sometimes the locational information delivered with them is inadequate, and the data does not align properly with other data you may have. Thus, in order to use some raster datasets in conjunction with your other spatial data, you often need to align it, or georeference it, to a map coordinate system. A map coordinate system is defined using a map projection (a method by which the curved surface of the earth is portrayed on a flat surface). When you georeference your raster dataset, you define its location using map coordinates and assign a coordinate system [6]. Georeferencing raster data allows it to be viewed, queried, and analyzed with other geographic data.

Therefore, when representing real-world features in a GIS, you need to reference the data describing them to the correct location on the earth's surface. This is called georeferencing. Georeferencing process is the process of establishing a relationship between the data displaying in GIS software and its real-world location. This is accomplished by using a coordinate system [7].

CAD drawings are usually much more accurate to a large scale than GIS data, but often use different coordinate systems than GIS. CAD drawing will have no recognizable coordinate system, which usually 
means the drawing uses an arbitrary, locally established point of origin $(0,0)$. Identifying a CAD drawing's coordinate system is very important for a clean transfer to GIS.

Must of the available remote sensing data (satellite images, map, aerial photographs, and GPS measurements) that have been in GIS researches and projects are projecting and georeferencing with respect to UTM-WGS84 coordinate system, therefore, this coordinate system was adopted in the present study to reproject the CAD data into new coordinate system. This coordinate system is very useful especially in determined the distance between any two points.

\section{CAD TO GIS Conversion Process}

\section{Preparing CAD data file}

In this section, the conversion process (adopted in this study) from CAD data file to the GIS georeferencing data file (or map) are explain. Starting from AutoCAD stage, in this stage it must be sure that all layers are turned on and the entire drawing extents are viewable. AutoCAD 2004 have been used to prepare the CAD feature layers that will be exported as (.dwg) format to the GIS software through the study. The adopted CAD drawing data is a sample composed of drawing Layers describes the total area of the Mosul University Campus. This drawing sample was editing by the Civil Engineering Department/University of Mosul.

Figure (1) illustrates the standard flow chart that can be as a reference guide in this study for the conversion process from CAD (.dwg) data file to GIS (.shapefile) georeferencing data file.

According to the purpose of the study, the entire CAD drawing will be transferring to ArcGIS9.1 software. But, there is a possibility that if there is not need to convert the entire layers (for some problems) and interest only in few layers of CAD data, it can select only the desired layers to transfer.

Before transferring the CAD drawing to GIS, it should be verify that all features are on their respective layers. Isolate each layer by turning all other layers OFF and see that there are no stray drawing elements or features that belong on other layers. Erase those elements that do not belong on the layer, or cut and paste them into their proper layer, this step is very important to obtain a good results through the working in the GIS software. The final output data file must be save in extension (.dwg) to be exported in next section by using ArcGIS9.1 software. Figure (2) shows the CAD.dwg of the study area as display in AutoCAD window. There is an other possibility to save the final output data in the extension (.dxf) which is used an intermediate format to export/import files from one software to another. This exchange format was created by Autodesk Co. to communicate AutoCAD with other software (GIS, Global Mapper, Erdas, MapInfo, etc) [5]. 
Spatial Georeferencing of the AutoCAD Map by Using GIS Software.

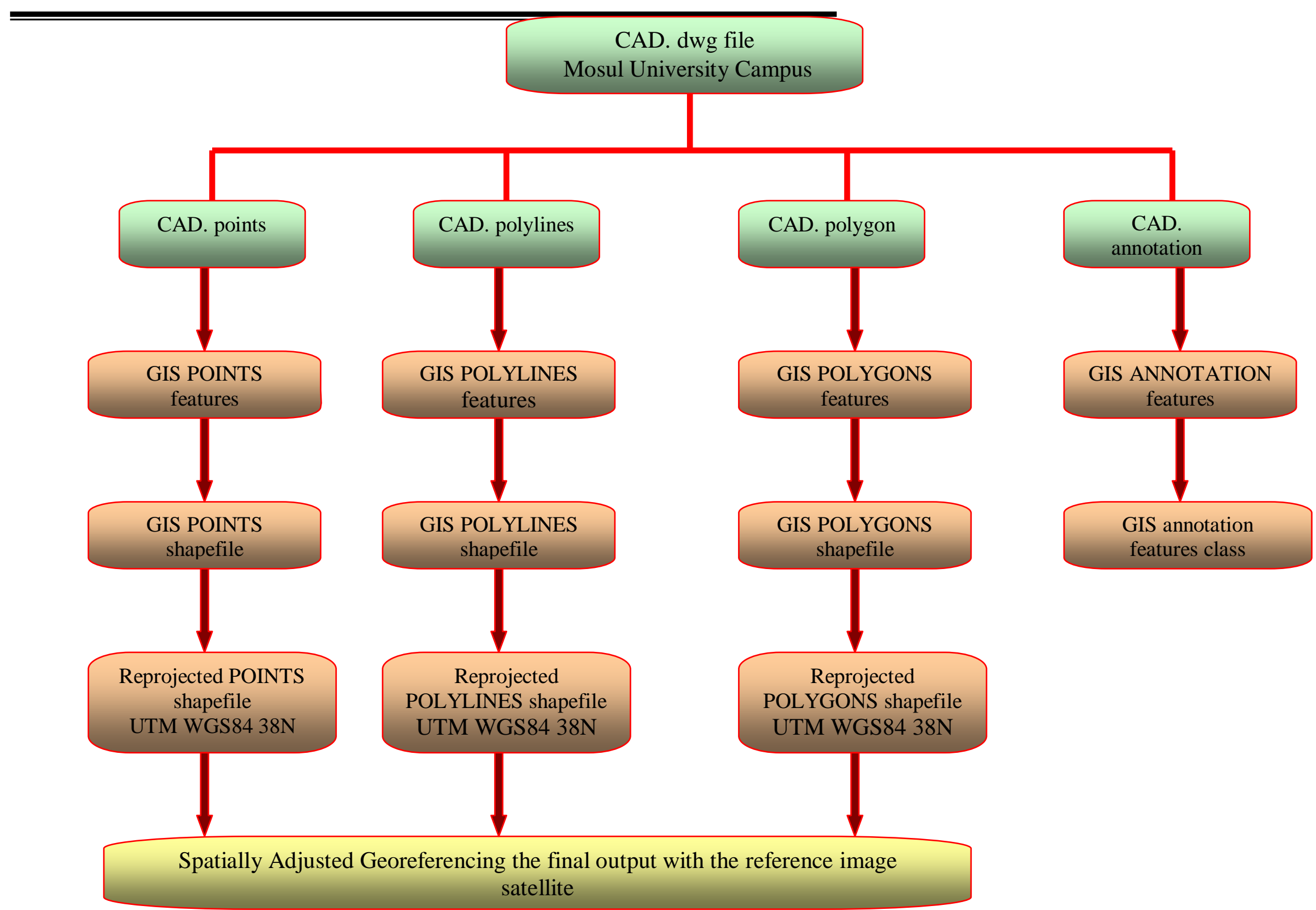

Figure (1): The flow chart of the conversion process

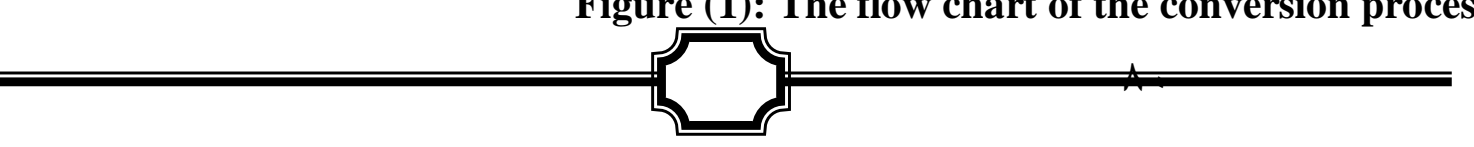




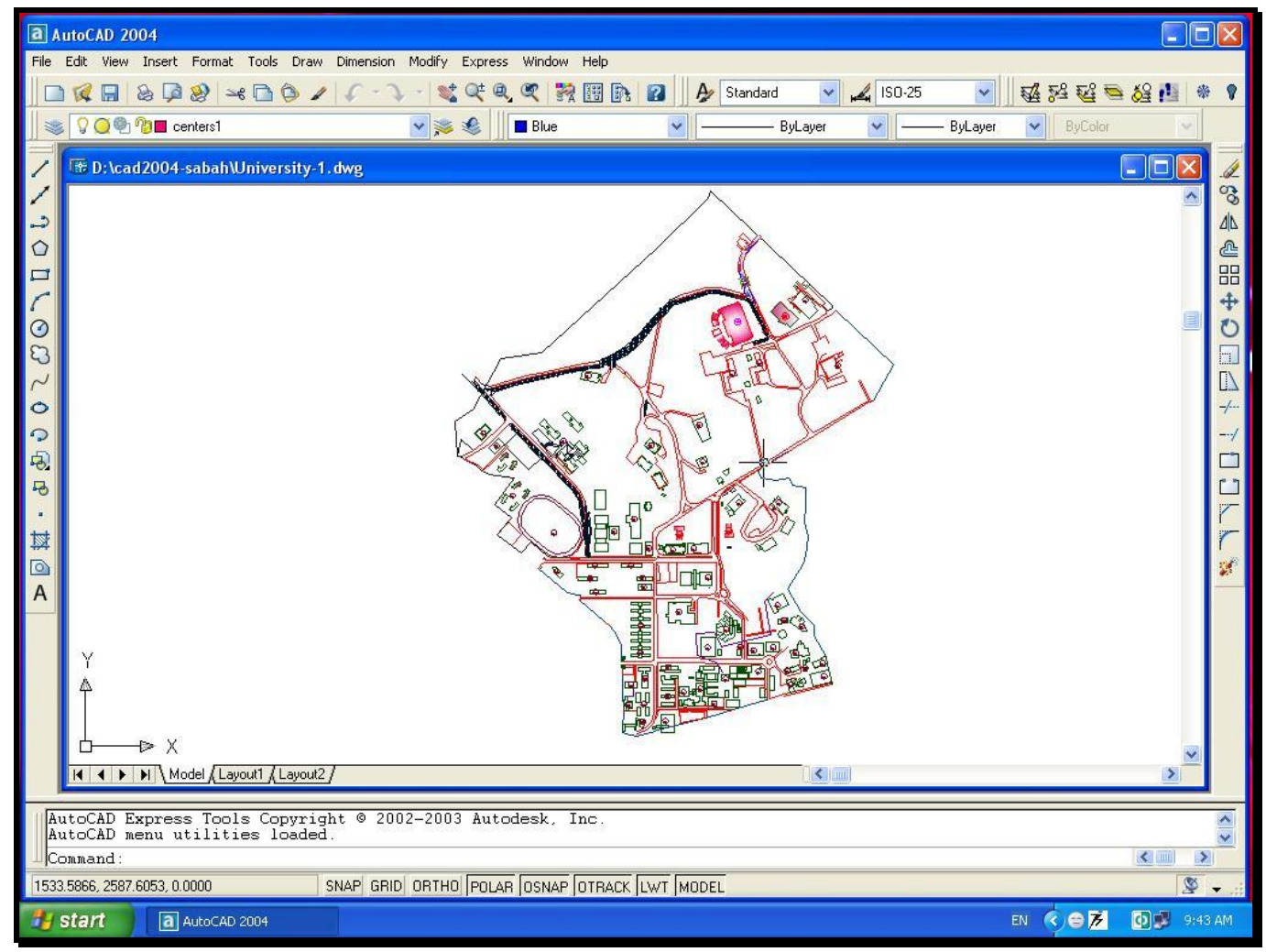

Figure (2): CAD.dwg file of the Mosul University

\section{Creating GIS Data From CAD File}

When a CAD drawing is imported into GIS, the GIS will interpret all elements into the four categories of GIS features: points, polylines, polygons, and annotation (text). Typically it will be desirable to further divide these shapefiles based on the original CAD layers. However, the adopted sample of the CAD.dwg file prepared in the last section was added to the ArcMap, using Add Data button (+). Through the working in the GIS stage, it must be note that when browsing for the CAD file, there are two files of the same name will appear. The first, displayed as a lightblue icon ( $)$ is a "CAD feature data set", which will convert the CAD file into point, polyline, polygon, and annotation (which is created from CAD text) objects. The second, displayed as a white icon (t) is the sample of CAD line drawing. In this file, text and polygons will read as linework only, and there will be no associated attributes for any objects [2].Therefore, the first file have been chosen to work with the ArcGIS software for the propose of producing shapefiles and georeferencing process.

When the blue CAD file was added to ArcMap, the file will be divided into data sets, based on the four types of GIS data: point, polyline, polygon, and annotation. Each data set (except annotation data 
set) will be associated with attribute table fields which will be created from the .dwg "CAD feature data set", these GIS fields included (Fid, Shape, Entity, Layer, Color, Linetype, Elevation, Thickness, Text, Font, Style, FontID, Height, Angle). These fields can be display in the attribute table of each layer through the right click on the desired layer on the Table Of Content (TOC) in ArcGIS9 window. Shapefiles can be created from point, polyline, and polygon data sets. The annotation set cannot be exported as a shapefile. Annotation must be exported as a feature class of a geodatabase.

Depending on the methodology of study, the CAD polyline and polygon data sets were only converted to GIS shapefiles (CAD point data is difficult to decipher, and CAD annotation is usually does not contain real data, only labeling).

In the ArcGIS9.1/ArcMap window, right click on the polyline and polygon data set and choose Data then Export Data, this order will export the polyline (or polygon) layer to primary shapefile with the same coordinate system defined by the CAD data (i.e.; resulting shapefiles will not yet have a defined coordinate system in GIS). Therefore the newly shapefiles are not compatible with GIS data. In order to align the newly created shapefiles to be proper with GIS data, it is necessary to have a coordinate system and projection defined in GIS.

\section{Reprojecting Shapefile to UTM_WGS84_38N}

As mention above the CAD sample applied here in this study have no defined coordinate system which may be means that drawing uses an arbitrary, locally established point of origin $(0,0)$, therefore the drawing sample must be reprojected in to new coordinate system in GIS for proper alignment with other GIS data. If there is no information on the coordinate system and datum from the drawing or the creators, compare drawing coordinates in CAD with GIS coordinates of the same area using a georeferenced image satellite (as doing in this study) or other dataset such as several control points collected and measured by GPS receiver in the same area that covered the study area [8]. This information was very necessary for the correctly align the data within GIS.

At the present time, most of the available GIS and remote sensing data in Iraq are spatially georeferencing with respect to the (UTM) coordinate system and datum World Geodetic System 84 (WGS84). The UTM is a specialized application of the Transverse Mercator projection [9]. In the UTM projection, globe is divided into 60 north and south zones, each spanning six degrees of longitude. The origin for each zone is its central meridian and the equator. Each zone has its own central meridian. The value given to the central meridian is the false easting, and the value assigned to the equator is the false Northing. A false Easting of 
500,000 meters is applied. A north zone has a false Northing of zero, while a south zone has a false Northing of 10,000,000 meters. Many countries use local UTM zones based on the official geographic coordinate systems in use. IRAQ country are covered with the zones $(37 \mathrm{~N}, 38 \mathrm{~N}, 39 \mathrm{~N})$ on the north side of the globe. Most of the Iraqi area are covered with the zone $(38 \mathrm{~N})$.

By displaying the properties of each created layer through the ArcGIS9.1/ArcMap, it founds that all the layers haven't a define coordinate system. In this case it must be define a coordinate system for each layer separately by using ArcGIS9.1/ArcCatalog.

The new polygon shapefile was preview in the ArcCatalog window as shown in figure (3).

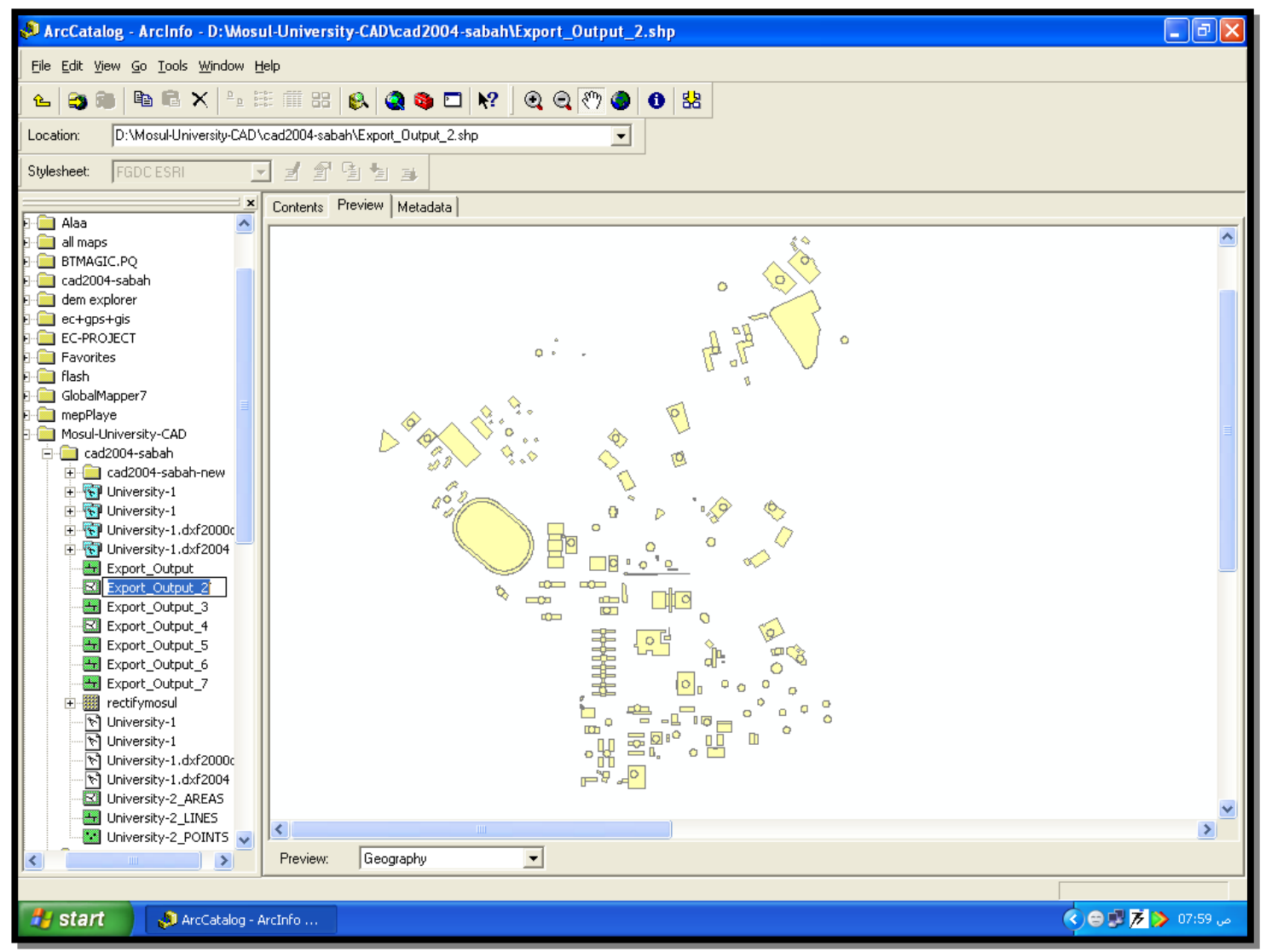

Figure (3): The polygon shapefile as previewed by ArcCatalog

To reproject the shapefile shown in figure (3), right click on the shapefile layer and click Properties. In the Spatial Reference tab click on Edit and select the coordinate system parameters that confirmed with those of the reference image satellite that covered the same area of the CAD drawing (Part of the Mosul University Campus). Figure (4) shows the IKONOS image satellite of the Mosul University Campus which have been used as a reference map in the spatial adjustment process. 


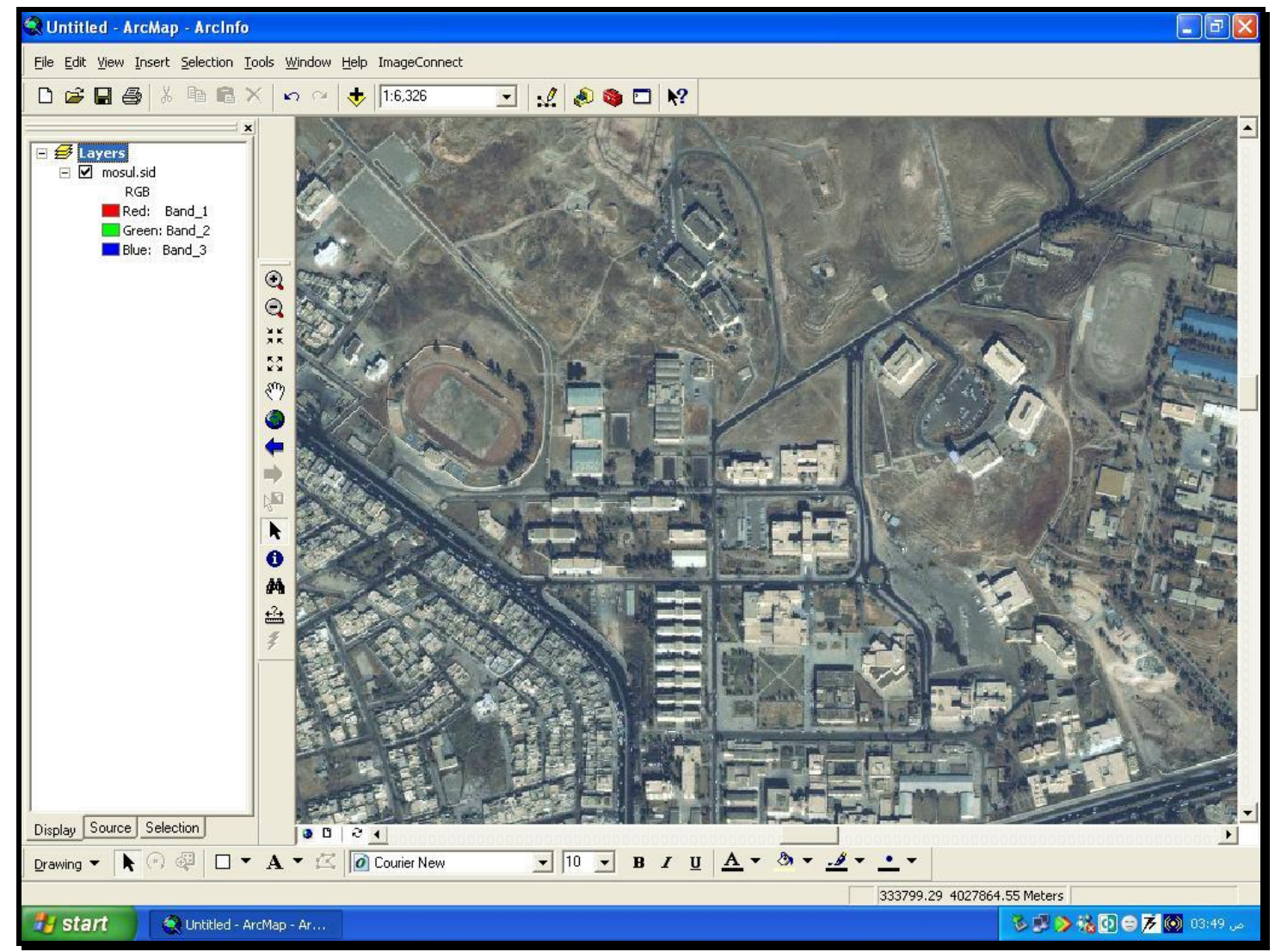

Figure (4): Image satellite of the Mosul University Campus

All the created shapefiles in this study were reprojected and rectified to the coordinate system of UTM_WGS84_38N. This coordinate system is already defined for the applied image satellite shown in figure (4).

Table (1) listed the parameters of the UTM coordinate system at the zone $(38 \mathrm{~N})$. Figure (5) demonstrates the projected UTM coordinate system parameters as shown in the ArcGIS9.0/ArcCatalog window.

Table (1): The Parameters of the UTM Coordinate system

\begin{tabular}{|c|c|}
\hline Parameters & Standard Values \\
\hline \hline False_Easting & $500000 \mathrm{mE}$ \\
\hline \hline False_ Northing & $0 \mathrm{mN}$ \\
\hline \hline Central Meridian & 45 Degree \\
\hline \hline Latitude of Origin & 0 Degree \\
\hline \hline Scale Factor & 0.9996 \\
\hline
\end{tabular}




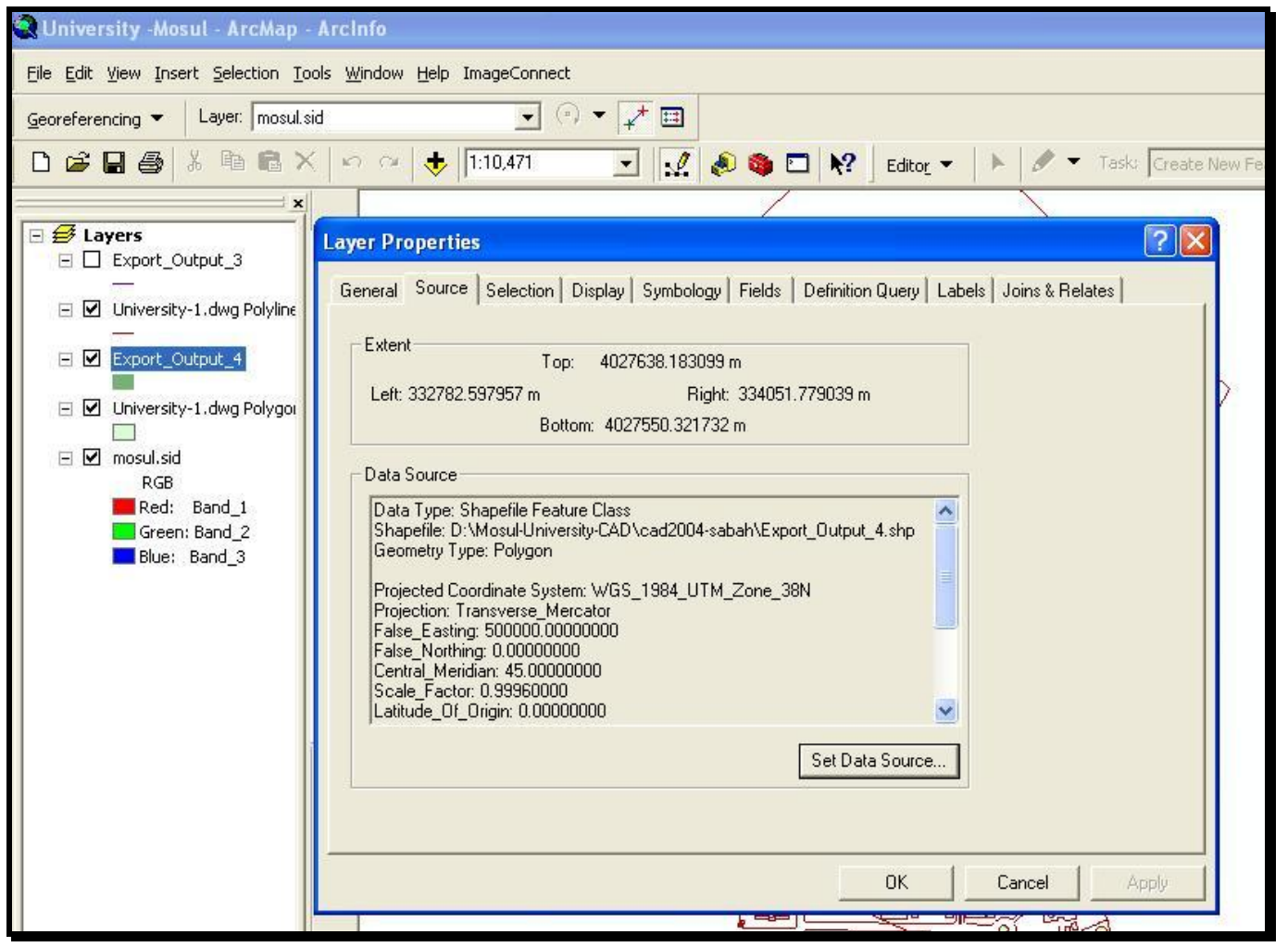

Figure (5): The parameters of the UTM_WGS84_38N

The final stage involved the aligning of the reprojected shapefiles with the reference image satellite shown in figure (5). This process was performed in a blank ArcMap project where the correctly georeferenced image satellite of the study area (which have been used as a planimetric base for georeferencing the newly created shapefiles) and the new projected polygon shapefile were added. In this process, there should be common elements visible in both polygon shapefile and the image satellite. These common elements will act as the control points for the adjustment process. In the spatially aligning process, the Editor and Spatial Adjustment toolbars must be activated. Then, the new displacement link was clicked on the strategic spot on the shapefile, the next one clicked was executed on the identical spot on the image satellite. In this study, four inserting links have been established to ensure the correcting align of both shapefile and image satellite. Figure (6) illustrates the four displacement links of the common control points between the polygon shapefile and the reference image satellite.

\begin{tabular}{|lllll|}
\hline E LINK-POLYGON - Notepad & & & 口 \\
\hline File Edit & Format View Help & & & \\
\hline 5 & 1450.644722 & 2458.341139 & 333720.417896 & 4027733.459078 \\
4 & 1137.098442 & 2127.179900 & 333411.119361 & 4027411.195933 \\
3 & 1549.288496 & 2320.944455 & 333825.986857 & 4027596.404637 \\
2 & 843.912856 & 2384.079865 & 333112.93347 & 4027657.523509 \\
& & & & \\
\hline
\end{tabular}

Figure (6): The four displacement links of the Spatially Adjustment process 


\section{Results and Discussion}

Spatially adjustment process will move a shapefile from its current coordinates to the correct coordinates by aligning control points in the shapefile with identical control points from a correctly projected image satellite or other dataset of the same geographic area. As mentioned above, before spatially adjusting the projecting of both polyline and polygon shapefiles must be define as UTM_WGS84. In this projected mode (coordinate system) the location of any points in the final output map will be identified by (x, y) coordinates on a grid. One specifies its horizontal position and the other its vertical position.In the UTM term, the two values are called the Easting (E) -coordinate and Northing $(\mathrm{N})$ coordinate.

However, through the working in the spatially adjustment process, it is found that alignment of the new polyline shapefile layer was not coincided with reference image satellite as done by polygon shapefile, this can be related to the basic CAD drawing properties of polyline layer which have not match with the line feature class that already defined in the GIS properties, and also may be to the applied method of the drawing. Therefore, the present study was focusing on the spatially alignment of the polygon shapefile layer which have been successfully adjusted.

Figure (7) shows the reprojected polygon shapefile created by the ArcGIS/ArcMap from the basis CAD polygon drawing (.dwg) file. The figure illustrates the buildings and the stadium inside the Mosul University campus. The projection of the polygon shapefile layer shown in figure (7) was defined as UTM_WGS84, this projection was fixed to the polygon layer through the GIS stage. Figure (8) shows the final result of the spatially adjustment process between the reprojected polygon layer and the reference IKONOS image satellite of the study area. The figure shows the relatively perfect coincidence between the polygon layer and the image satellite. The more accurate coincidence can be resulted from the more displacement links of the control points between the layer and the reference image satellite. From the figure (8), it can be shown that some CAD drawings were out off the original locations that explained in the image satellite and another drawings is unnecessary. This drawing mistakes can be editing and correcting by the ArcGIS editing functions.

The applied conversion techniques, will be able the planner to make a perfect correction for his CAD drawings with respect to the reference image satellite by using the ArcGIS software.

For the spatially comparison purpose between the coordinate system readout of the ACD drawing before the spatially conversion process and after the applying of the complete conversion process through the ArcGIS/ArcMap and ArcGIS/ArcCatalog windows, figure (9) 
explains the difference in the readout of the coordinate system between the two format (CAD and GIS ) for a certain spot locate in the stadium. It was show that the coordinate system of the spot in the CAD window give the readout of $(x=856.2648, y=2356.7632)$ according to the locally establish point of origin $(0,0)$ as shown in figure $(10 \mathrm{a})$, while the readout of the same spot (after reprojected and spatially adjustment process) in the reference image satellite was $(\mathrm{E}=333107.69, \quad \mathrm{~N}=4027641.98)$ according to the worldwide define projected system (UTM_WGS84_38N). In the second coordinate system, it was easy to make any link and data exchange with a common remote sensing data (image satellite, aerial photography, radar image, GPS dataset, ect.) with the same projection. At this stage, all the geoprocessing operations can be applied through the ArcGIS software. Figure (10), shows the resulted layout of the coincidence map between the new CAD drawing and the Image satellite with the identified coordinate system in UTM mode (E, $\mathrm{N}$ ), this map can be as dependent geographical reference for any geodetic and survey application in the Mosul University Campus.

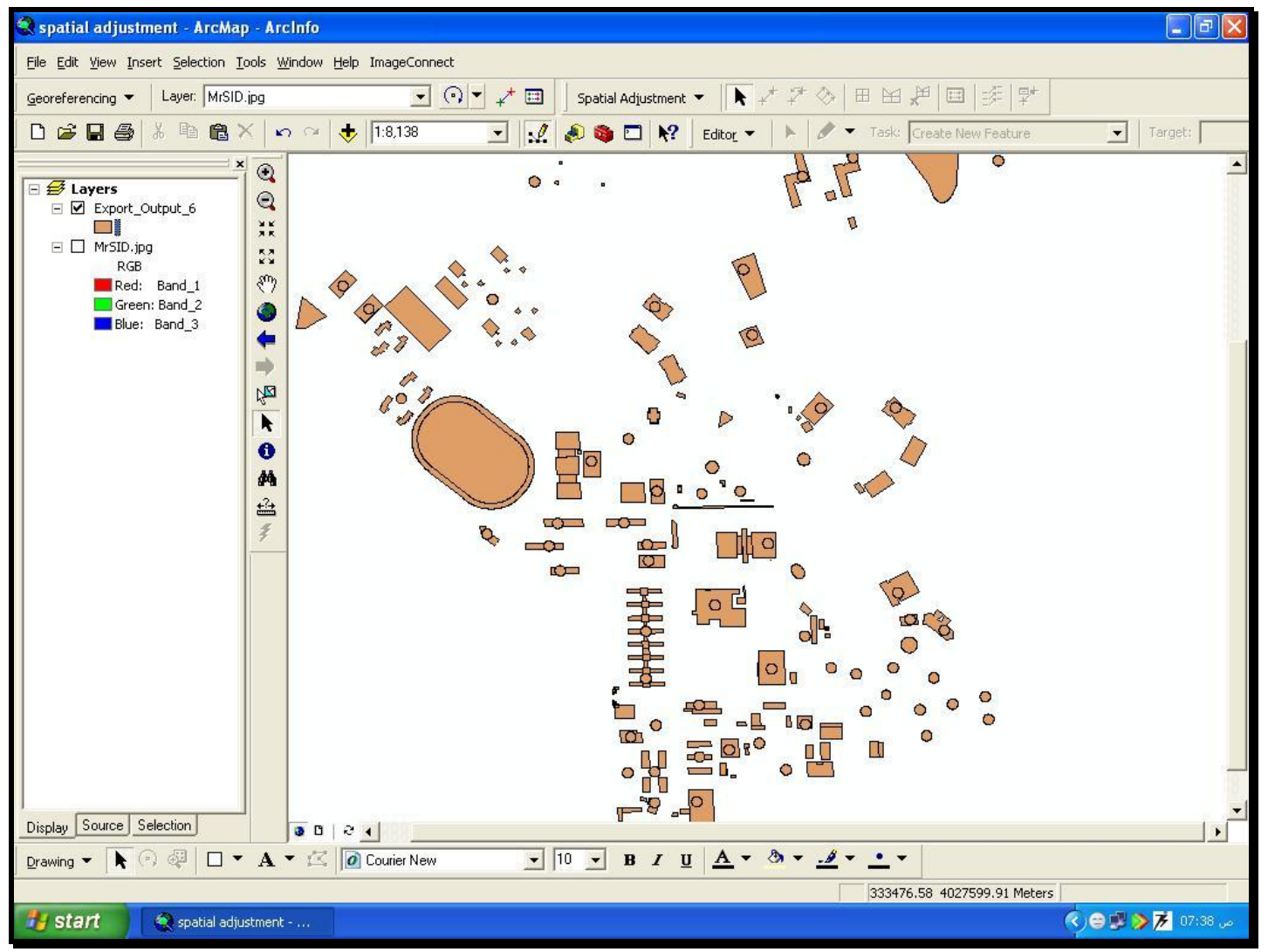

Figure (7): The polygon shapefile feature class 
Spatial Georeferencing of the AutoCAD Map by Using GIS Software.

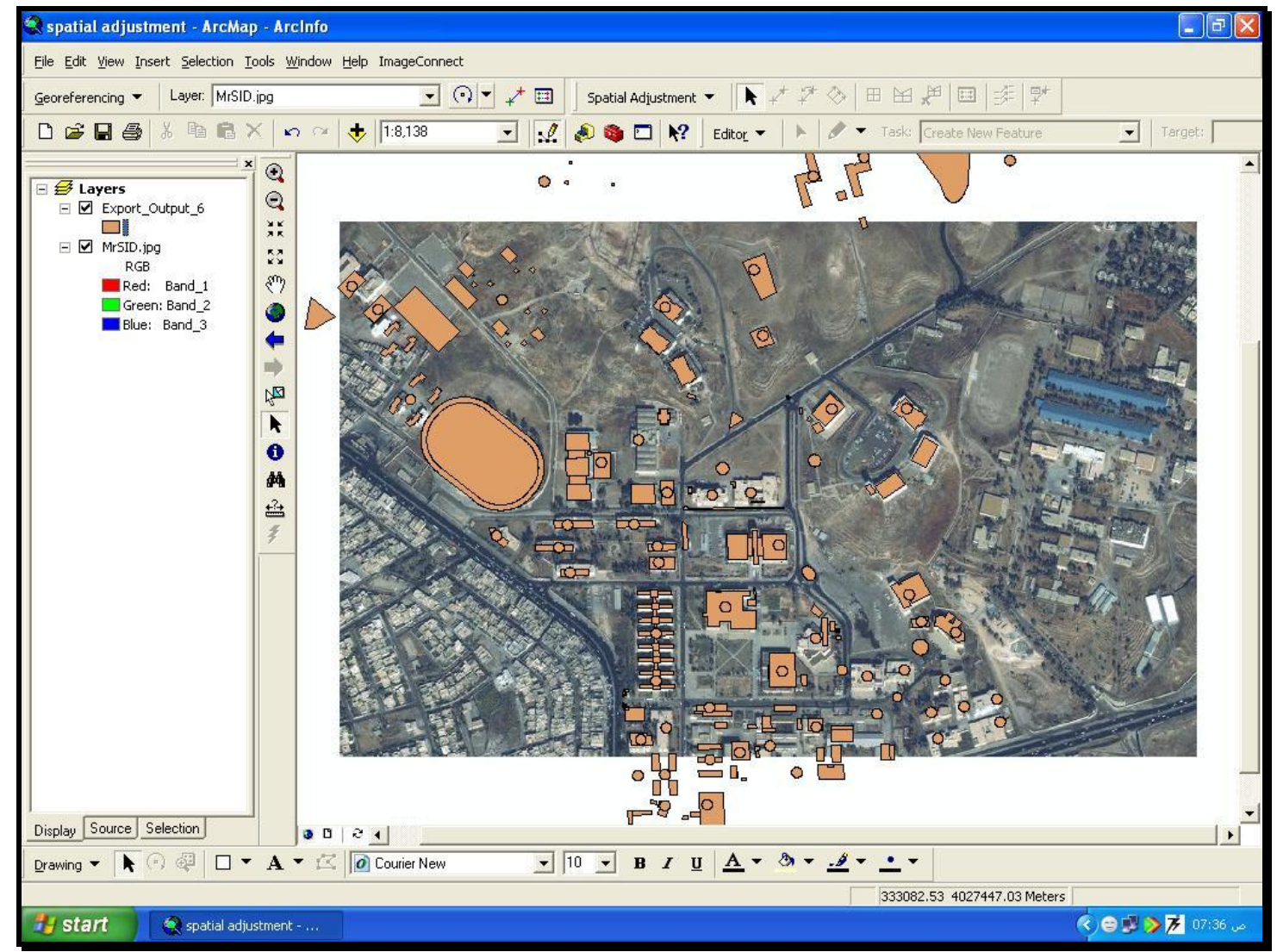

Figure (8): Spatially adjustment between polygon layer feature class and the IKONOS image satellite

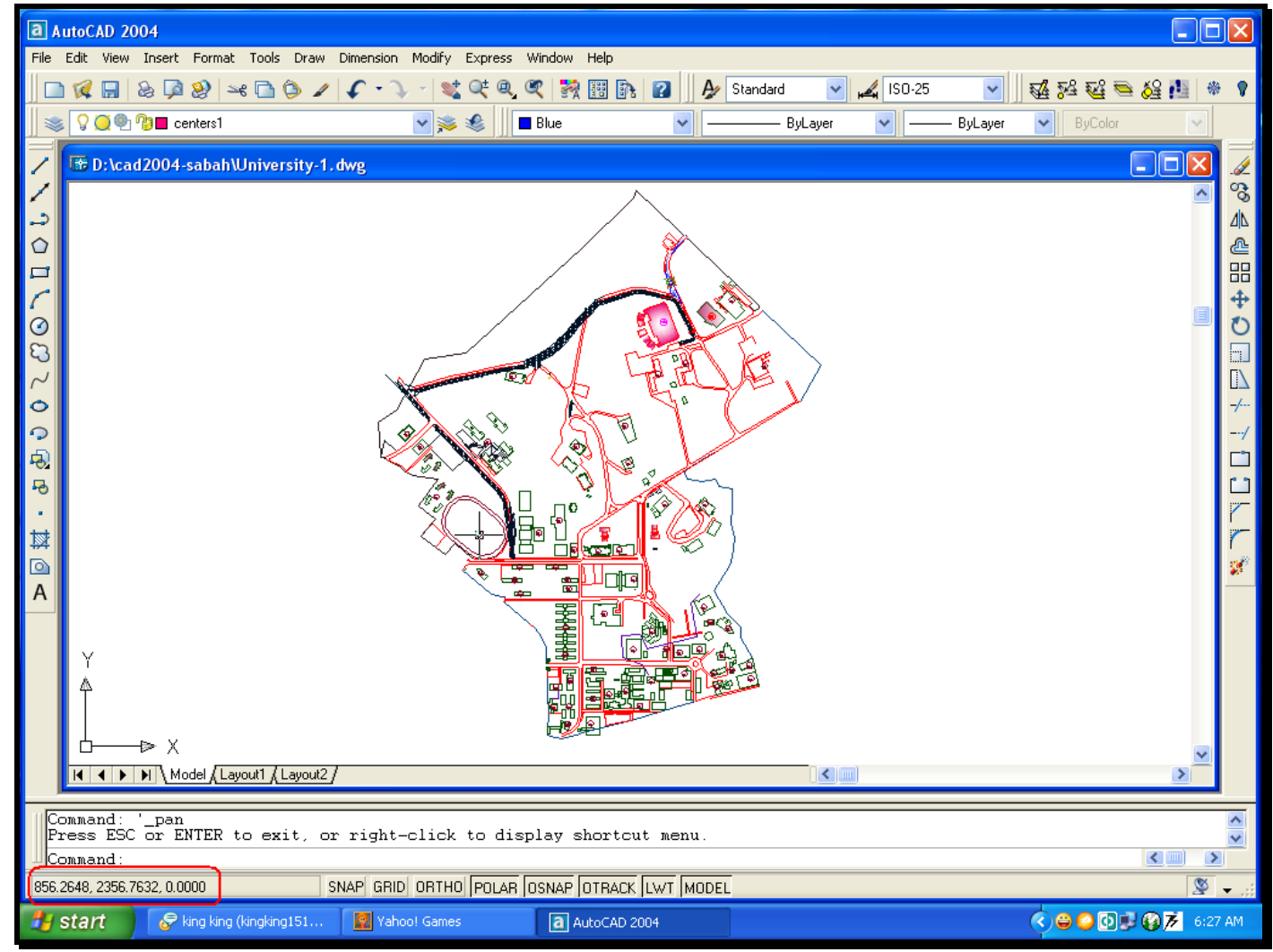

Figure (9a): The readout of the CAD drawing in the AutoCAD window 


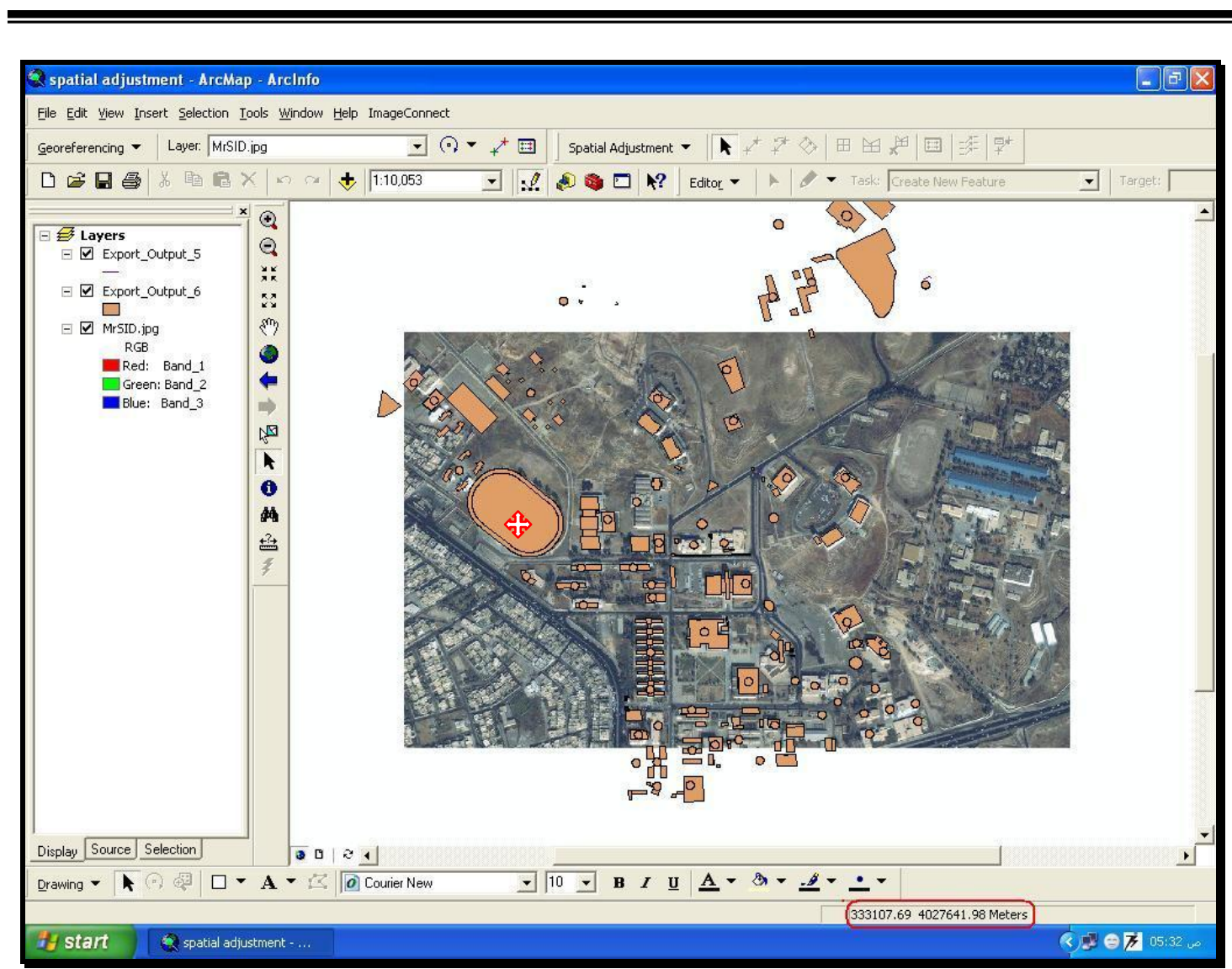

Figure (9b): The readout of the new reprojected CAD drawing in the GIS window

Figure (9): Compare coordinates of identical spot in CAD and GIS 
Spatial Georeferencing of the AutoCAD Map by Using GIS Software.

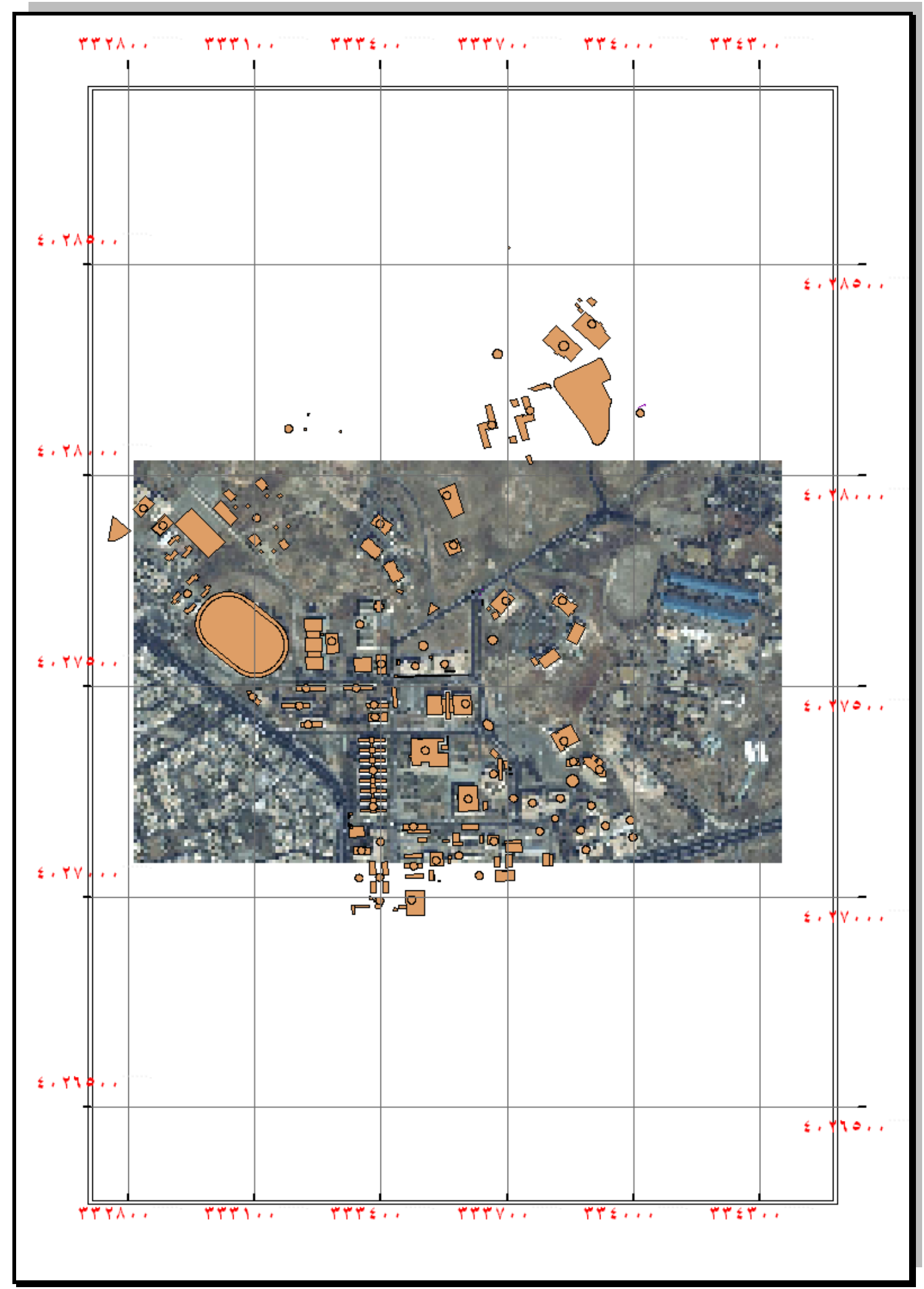

Figure (10): Spatially georeferencing map of both reprojected polygon CAD drawing and IKONOS image satellite 


\section{Conclusion}

The most interesting purpose in our study is create a spatially georeferencing GIS shapefiles from CAD drawings of unknown coordinate system. Then, the resulted shapefiles have been aligned to the reference image satellite of the study area with the concept of spatially displacement.

From the results of the applied CAD/GIS conversion methodology, it can be conclude that, this type of approach is an efficient and useful way to perform CAD conversion to GIS data when there is a direct correlation between CAD entities and GIS features. Also, to ensured the high level accuracy of the spatially adjustment process for the shapefile layer with the reference image, It is prefer to use more control points links which will improve the accuracy of the alignment process.

Finally, the GIS view of the CAD data can be used as input to GIS tools, functions, and processes to codify rules-based semantic translation in the GIS language, and then it is possible to compare both data and analyze this information with the help of the thematic maps.

\section{References}

1) Keith C. C., 1999, "Getting started with Geographic information system“, 2nd edition, Prentice-Hill, Inc., 338p.

2) Kasianchuk, P., Taggart, M., 2004, "Introduction to ArcGIS-I", Course version 4.2, ESRI, Redlands, USA

3) Ahmedi M., 2003, "Crime Mapping and Spatial Analysis", Thesis submitted to the International Institute for Geo-information Science and Earth Observation, ENSCHEDE, THE NETHERLANDS, 64p.

4) "ESRI Shapefile Technical Description", An ESRI White Paper-July 1998, available at www.esri.com, accessed at: 2/Jan/2008, 34p.

5) Torralba I. S., 2006, "The Historical Hungary of 1914: A MapInfo Based Project", Thesis submitted to Eotvos Lorand University, HUNGARY, 45p.

6) Rizos C., 1999, "Principle and Practice of GPS Surveying", The University of New South Wales, Sydney, Australia, available at: www.gmat.unsw.edu.su/snap, accessed at: 13/jan/2008.

7) Davis, E. B., 2001, "GIS: A visual Approach', $2^{\text {nd }}$ Edition, Onword Press, Canada, 438p.

8) Ali S. H, Al-Banna R. G., Al-Alaaf A. A., 2007, "Using of Geographical Information System and Global Positioning System to Establish Coordinate System for the Remote Sensing Data", Journal of Education and Science, vol.14, no.1, pp.1-11.

9) Christopher, B., J., 1999, "Geographical Information Systems and Computer Cartography", Addison Wesley Longman Limited, 318p. 